\title{
The role of voluntary organizations in astronomy popularization: a case study of Khagol Mandal
}

\author{
Aniket Sule ${ }^{1,5}$, S. Joshi ${ }^{2}$, H. Joglekar ${ }^{3}$, A. Deshpande ${ }^{3,4}$, M. Naik ${ }^{3}$ \\ and S. Deshpande ${ }^{3,5}$ \\ ${ }^{1}$ Astrophysikalisches Institut Potsdam, Potsdam, Germany \\ ${ }^{2}$ Jodrell Bank Observatory, University of Manchester, Macclesfield, UK \\ ${ }^{3}$ Khagol Mandal, Mumbai, India \\ ${ }^{4}$ Society for Applied Microwave Electronics Engineering and Research, Mumbai, India \\ ${ }^{5}$ Homi Bhabha Center for Science Education, Mumbai, India \\ email: aniket.sule@gmail.com
}

\begin{abstract}
We present a case study of "Khagol Mandal," a voluntary organization primarily based in Mumbai, India. In the 20 years since its inception, Khagol Mandal has given more than 1000 public outreach programmes. The volunteers strive to go beyond amateur level by means of various study tours, astronomical experiments and workshops. These activities have inspired a number of students to take professional astronomy careers. With a volunteer force, probably largest in India or even south Asia, Khagol Mandal is well poised to take advantage of the archival data of large telescopes. With a little guidance from senior researchers, organizations like Khagol Mandal can provide a solution to the ever-increasing need for manpower for ancillary science from these large-scale facilities.
\end{abstract}

Keywords. Astronomy education, astronomy popularization

\section{The need for voluntary organizations in astronomy popularization}

In India the population density is such that it overwhelms the education and public outreach potential of most astronomical research institutions, who are hard pressed to cover all the schools and colleges in their area. Organizations of amateur astronomers can extend the outreach potential of astronomy research institutions in several ways by conducting various programmes such as exhibitions, slide shows and sky observations to popularize astronomy. At the same time, with their programmes, these organizations can attract young students and guide them to a research career in astronomy. We present the case study of an organization run by amateur astronomers by giving a detailed account of the formation and activities of the organization.

\section{Khagol Mandal}

Khagol Mandal (www.khagolmandal.com) is a voluntary organization, working in the field of astronomy, primarily based in Mumbai. In the regional language, Marathi, 'Khagol' refers to the celestial sphere, while 'Mandal' refers to a group of people involved in an activity. Khagol Mandal was started by a group of astronomy enthusiasts from Mumbai in 1986, the year when the Halley's comet reappeared in the sky. Twenty years down the line, Khagol Mandal operates from various units in and around Mumbai, with over 1000 members. The main unit is based in Mumbai while the other 4 units are at Badlapur, Dombivali, Thane and Nashik. These units have their own libraries dedicated 


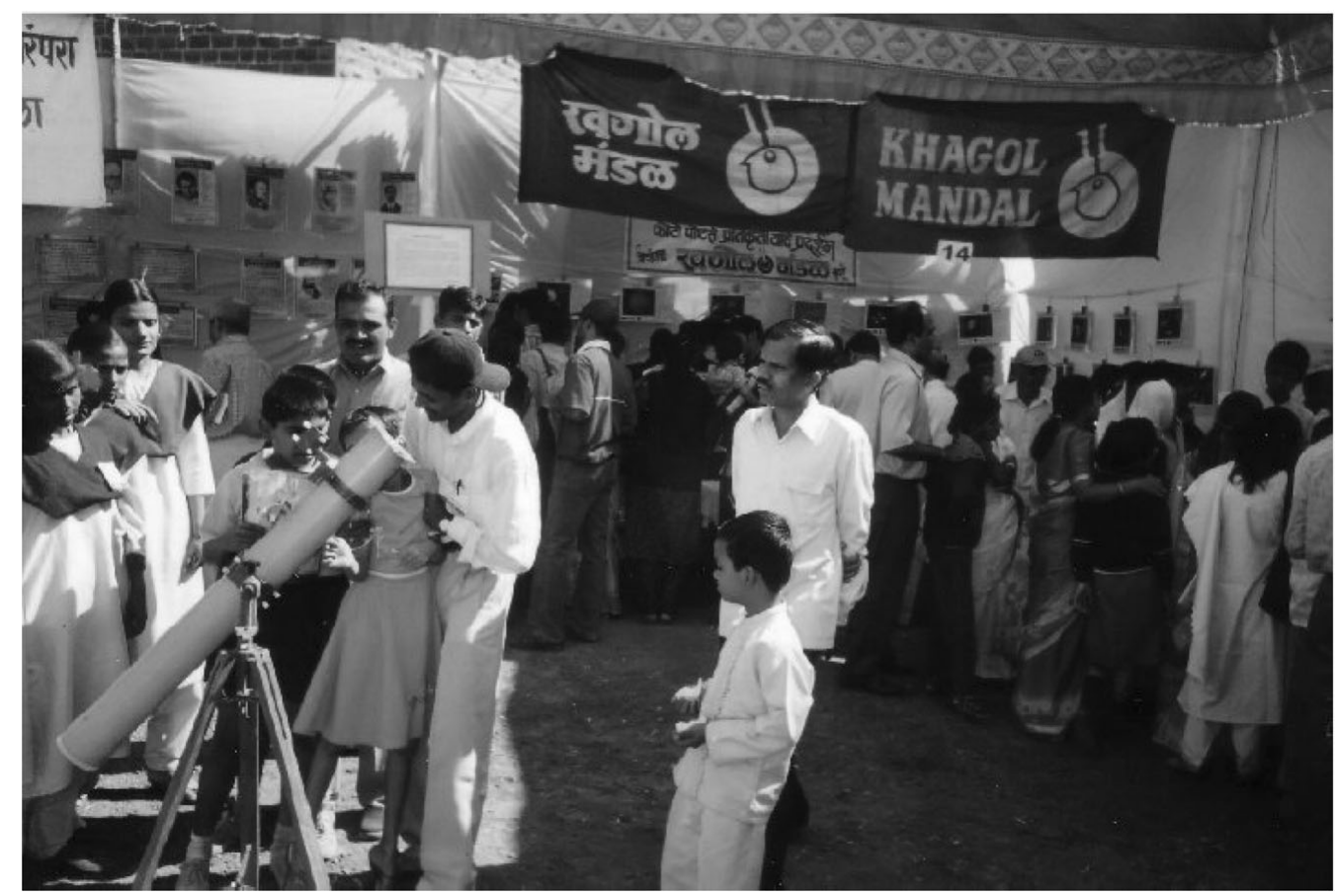

Figure 1. Response from all age groups for an exhibition by Khagol Mandal. Credit: Sameer Kadam

to different aspects of astronomy, arrange regular lectures on various astronomical topics for their members and train them for various public outreach activities.

\section{Activities of Khagol Mandal}

Khagol Mandal organizes various programmes for the general public on a regular basis, such as sky observation programmes, exhibitions, and astronomy-related talks in schools, colleges, clubs and community centres.

\subsection{Weekly meetings and library}

At each unit the members gather on a particular day of the week. On that day, lectures, discussions and question-and-answer sessions are conducted. The library opens on that day and the members can read and/or borrow the books. There are about 650 books available at the library of the main unit, while the libraries of the other units contain about 600 books in total.

\subsection{Sky observation programmes}

Khagol Mandal organizes regular overnight sky observation programmes at Vangani, a sleepy village on the outskirts of Mumbai, the city where the main unit is based. Avoiding the cloud-covered monsoon months, the programmes take place once a month from October to May. Programmes are conducted on Saturday nights which are close to the new moon. These overnight programmes are open to the general public. The programme consists of an introduction to telescopes, the use of constellations to find directions, identification of constellations visible with their main points high-lighted and observations of solar system and deep sky objects through telescopes. The programme also involves 


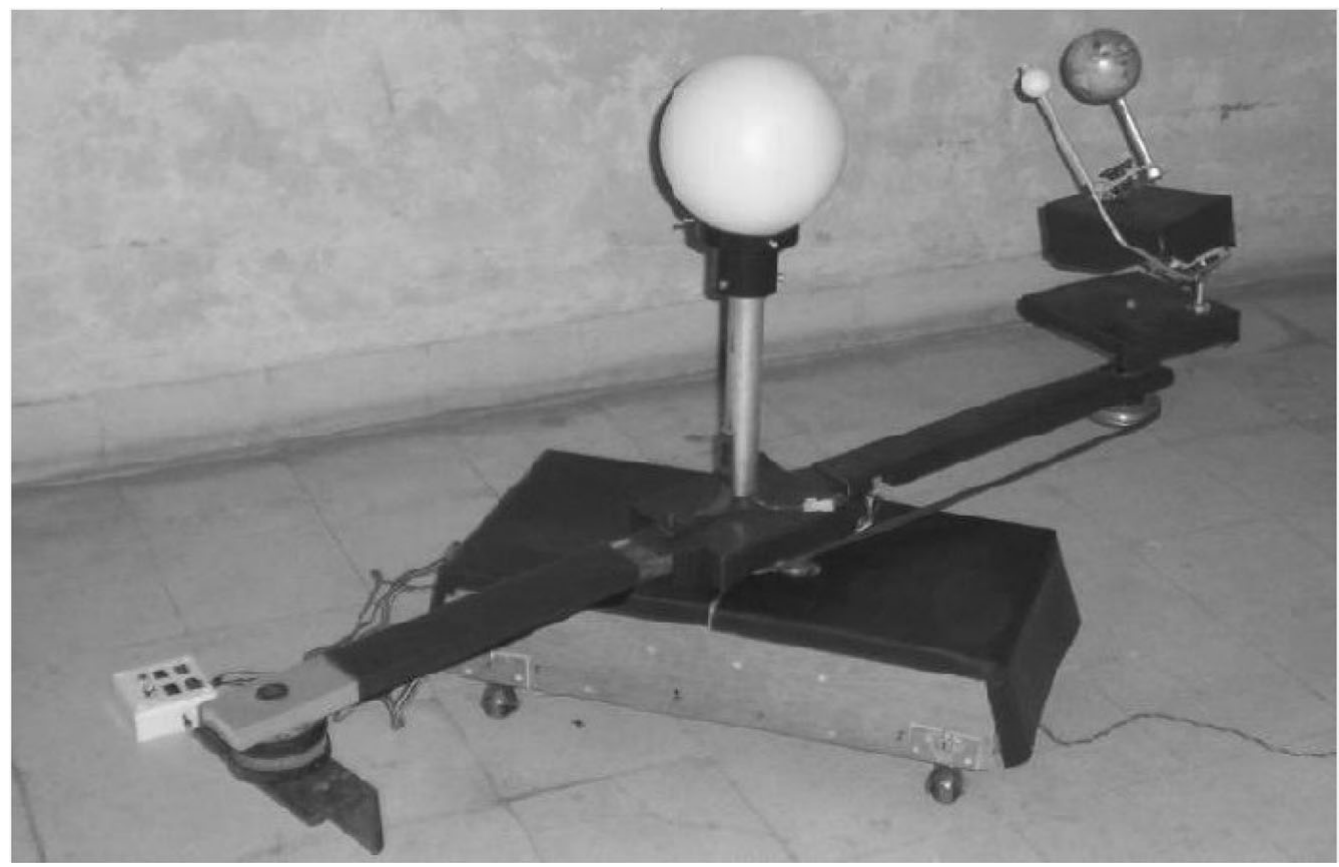

Figure 2. Model of the Sun-Earth-Moon system that demonstrates phenomena like the seasons, eclipses, precession, etc. Credit: Abhir Joshi

a slide show (see below) on one of the various astronomy topics. A question-and-answer session of a highly technical nature is always conducted during the programme where the experts from Khagol Mandal give scientific answers to the questions asked by the audience. Sometimes parallel programmes are conducted in the regional language Marathi and in English. Apart from the overnight sky observation programmes, short duration programmes are conducted as per the requirements of schools, colleges, clubs or people of residential complexes on their premises.

\subsection{Exhibitions}

Khagol Mandal has a collection of about 100 A0 and A3 sized charts on topics such as the solar system, Messier objects, and the Lonar meteor crater, which are the made by the members of Khagol Mandal. Khagol Mandal puts up these charts either in the independent programmes arranged by Khagol Mandal itself or in the programmes arranged by other science popularization bodies (see Figure 1). The exhibitions are also put up in education institutions at their request. Though the charts are self-explanatory the volunteers of Khagol Mandal are present to share the information with the viewers.

\subsection{Slide-shows}

Khagol Mandal has a collection of about 650 slides which cover a wide range of topics such as stellar evolution, the solar system, Saturn, Jupiter, extra-terrestrial life, eclipses, transits, occultations and comets. The slide shows are conducted independently or as part of other programmes of Khagol Mandal. The members conduct slide shows at various public places and at educational institutions. 
Table 1. List of conferences and workshops in which volunteers of Khagol Mandal have participated, the activities they performed and the topics they covered

\begin{tabular}{|c|c|c|}
\hline Year & Conference/Workshop & Activities/Topics \\
\hline 1987 & Astronomy conference, Thane, India & General astronomy \\
\hline 1993 & $\begin{array}{l}\text { Conference on Bhaskaracharya's } 800^{\text {th }} \\
\text { anniversary, Mumbai, India }\end{array}$ & Mercury transit \\
\hline 1996 & Astronomy conference, Aurangabad, India & $\begin{array}{l}\text { Exhibition, sky observation, slide shows, } \\
\text { Lonar Crater discussion }\end{array}$ \\
\hline 1998 & Astronomy conference, Nashik, India & Exhibition, sky observation, slide shows \\
\hline 2002 & Lonar Crater Conservation Conference & $\begin{array}{l}\text { Physics of Lonar Crater, } \\
\text { Introduction to Lonar }\end{array}$ \\
\hline 2003 & $\begin{array}{l}\text { International Conference for Science } \\
\text { Communicators }\end{array}$ & $\begin{array}{l}\text { Roles of amateurs, astro-photography } \\
\text { and planetary transits }\end{array}$ \\
\hline 2005 & Astronomy conference, Pune India & Exhibition and sky observation \\
\hline 2005 & $\begin{array}{l}\text { Konkan Marathi Sahitya Parishad } \\
\text { (conference for Marathi literature) }\end{array}$ & Exhibition \\
\hline 2005 & Astronomy conference, Goa, India & $\begin{array}{l}\text { Computers in astronomy, } \\
\text { Observational astronomy, } \\
\text { Exhibition, sky observation, slide shows }\end{array}$ \\
\hline
\end{tabular}

\subsection{Basic and advance courses in astronomy}

Volunteers of Khagol Mandal conduct basic and advanced courses in astronomy almost every year. These courses are offered to the general public. The lectures are conducted by the experts of Khagol Mandal and by invited professional astronomers and physicists.

\subsection{Study tours, workshops, conferences and special event programmes}

On the occasion of rare astronomical events, Khagol Mandal organizes extensive public outreach campaigns as well as study tours, like the tours during the total solar eclipses (TSE) of 1995 and 1999, and public telescope observations at popular city locations during the Mercury and Venus transits. The Venus transit in 2002 was observed by over 23000 people through the telescopes set up by Khagol Mandal at various locations. The annual public outreach of Khagol Mandal exceeds 15000 people. The star party at Vangani on the day of the Leonid meteor storm in 1999 was attended by roughly 10000 people, presumably a record in at least the south Asian region. From time to time volunteers of Khagol Mandal participate in various conferences (see Table 1).

\subsection{Publications}

Since its inception 20 years ago Khagol Mandal has been publishing a bulletin called Khagol Varta (which means 'Astronomy News'). This Marathi bulletin started as a quarterly magazine. It soon became bimonthly and from 2002 it became monthly. The bulletin covers the latest astronomy news and the activities of Khagol Mandal. Since 2004 Khagol Mandal has also published an English quarterly bulletin called Vaishwik (which means 'related to the Universe'). Table 2 lists the other publications of Khagol Mandal.

\subsection{Astronomy research}

Most of the active volunteers are students and young professionals with a basic training in scientific methodologies. A good number of members have also chosen research careers in astronomy and astrophysics for themselves. Khagol Mandal encourages its members 
Table 2. List of publications of Khagol Mandal

\begin{tabular}{lll}
\hline Year & Title \\
\hline Since & 1986 & Khagol Warta (monthly bulletin published in Marathi) \\
& 1986 & Khagol Parichay, Khagol Prakashan, Mumbai, India, 1st edition \\
1995 & Totality (special bulletin on the occasion of TSE 1995) \\
1999 & Khagras Khagol Prakashan, Mumbai, India, 1st edition \\
2001 & Tarangan, Pradeep Nayak, Khagol Prakashan, Mumbai, 1st edition \\
2002 & Care Lonar, Kardile et al. Khagol Prakashan, Mumbai, 1st edition \\
2002 & Lonar Vivar, Khagol Prakashan, Mumbai, 1st edition \\
Since & 2004 Vaishwik (quarterly bulletin published in English) \\
& 2005 Khagol Parichay, Khagol Prakashan, Mumbai, India, 2nd edition \\
& 2006 Astronomy for amateurs, Khagol Prakashan, Mumbai, India, 1st edition \\
\hline
\end{tabular}

to plan and participate in various projects related to astronomy. During TSE'95 and TSE'99, members participated in various experiments including spectrography of the solar corona. The meteor crater at Lonar, India is frequently visited by members to study rock and microbe samples from the area. Khagol Mandal also arranged the 'Lonar Crater Conservation Conference' in 2002 where topics like near-Earth objects, Lonar Crater: is it volcanic?, Physics of craters, Archeology of Lonar Crater, Flora and fauna of Lonar Crater, and Water of Lonar Crater were presented. Some members have been developing low-cost but efficient tools to demonstrate astronomical events, otherwise difficult to visualize (see Figure 2). With a large and semi-skilled volunteer force at its disposal, Khagol Mandal can surely undertake research projects that involve a huge amount of man-hours, if it works under the guidance of senior researchers.

\section{Discussion}

Khagol Mandal can share the burden of public outreach with the professional astronomical community thorough its extensive public programmes and by publishing bulletins (such as Khagol Varta and Vaishwik) and books on basic astronomy. It can provide a semi-skilled workforce of volunteers who want to get a flavour of research and are capable of simple data analysis tasks. It can attract new students to astronomy by offering them the opportunity to work on research projects as a part of their hobby. Examples of such projects include:

- Determination of the extent of the bulge and disk of Milky Way by multi-colour stellar photometry from HST archives;

- Data mining in huge sky surveys, e.g. obtaining photometric red shifts for each object in the Hubble Deep Fields;

- Extensive surveys of archives for objects like gravitational lenses and novae, which may have missed detection.

Such projects can be performed as pilot surveys for professional astronomers. 